\title{
UWB Passive Navigation in Indoor Environments
}

\author{
(invited paper)
}

\author{
Stefania Bartoletti \\ ENDIF, University of Ferrara \\ Ferrara, Italy \\ stef.bartoletti@gmail.com
}

\author{
Matteo Guerra \\ ENDIF, University of Ferrara \\ Ferrara, Italy \\ matteo.guerra@student.unife.it
}

\author{
Andrea Conti \\ ENDIF, University of Ferrara \\ Ferrara, Italy \\ a. conti@ieee.org
}

\begin{abstract}
Localization and navigation of passive target objects play a key role in many important applications. An interesting solution for passive localization and navigation is given by monostatic wireless sensor radar (WSR) networks. In this context, ultrawide band (UWB) radar provide fine delay resolution enabling high accuracy localization also in harsh environments such as indoor. We present a mathematical framework for analysis and design of passive navigation based on UWB monostatic WSRs that relies on environment propagation and time-of-arrival estimation characterized by network experiments. A case study where a UWB monostatic WSR network is deployed to infer the position of moving target objects is considered. In particular, Bayesian navigation based on particle filters implementation is analyzed and the role of mobility model for inferring target position is shown.
\end{abstract}

\section{Categories and Subject Descriptors}

C.2.1 [Computer-Communication Networks]: Network Architecture and Design-Wireless communication

\section{General Terms}

Algorithms, Design, Performance

\section{Keywords}

Passive navigation, TOA estimation, UWB, particle filters, mobility models.

\section{INTRODUCTION}

Network localization and navigation is an emerging paradigm enabling new important applications in various sectors such as medical, industrial, and military [20]. Depending on whether the objects to be localized infer their positions actively exchanging messages or passively backscattering signals, the navigation process is referred to as active or passive,

Permission to make digital or hard copies of all or part of this work for personal or classroom use is granted without fee provided that copies are not made or distributed for profit or commercial advantage and that copies bear this notice and the full citation on the first page. To copy otherwise, to republish, to post on servers or to redistribute to lists, requires prior specific permission and/or a fee.

ISABEL '11, October 26-29, 2011 Barcelona, Spain

Copyright 2011 ACM 978-1-4503-0913-4/11/10 ...\$10.00. respectively $[8,18]^{1}$

The provision of location-awareness in cluttered environments (e.g., indoor) is challenged by multipath, line-of-sight (LOS) blockage, and excess delay propagation through materials. Ultrawide bandwidth (UWB) technology $[21,22,24]$ can provide accurate localization $[10,18,19]$ in such cluttered environments due to its ability to resolve multipath, penetrate obstacles, and provide accurate time-of-arrival (TOA) meaurements $[6,15,23]$.

Monostatic wireless sensor radar (WSR) networks extend the classical concept of single monostatic radar for passive detection, localization, and navigation of target objects [3, 12, 16, 25]. UWB based WSRs networks can provide high localization and navigation accuracy in harsh environments, such as indoor [2]. A clear understanding on how network setting and environment propagation affect the performance can be obtained through characterization of these aspects based on network experiments [5]. Several algorithms can be used to perform navigation from TOA measurements. In particular, Bayesian navigation based on particle filters (PFs) and mobility models provide solution to inference target object position.

In this paper, we present a mathematical framework for analysis and design of passive navigation. A UWB monostatic WSR network is considered and the framework accounts for the effects of network setting, obstructed line-ofsight (OLOS) propagation conditions, TOA estimation, and mobility models for Bayesian navigation algorithms. We model TOA estimation accuracy based on measurements collected in network experiments. A Bayesian navigation algorithm with PF implementation is considered which combines a prior knowledge based on mobility models with perception models and UWB ranging measurements. A case study in indoor environment is considered, where a UWB monostatic WSR network is deployed to infer the position of a target object moving on a trajectory by means of two different mobility models.

The remainder of the paper is organized as follows: Section 2 describes the WSR network model and Section 3 discusses the navigation algorithm considered. In Section 4 results on the case study considered are presented and in Section 5 some conclusions are given.

\section{WIRELESS SENSOR RADAR MODEL}

In a monostatic WSR network, $N$ nodes are involved in localization and navigation of a target object moving within

\footnotetext{
${ }^{1}$ Hybrid active and passive solutions can also be considered (see, e.g., [17]).
} 
a surveillance area (SA). We denote with $\mathbf{x}_{\mathrm{R}}^{(i)}$ the vector of $i$ th radar position coordinates (static) and with $\mathbf{x}_{k}$ the target position (dynamic) at time index $k$. Each radar transmits a sequence of pulses at a given pulse repetition frequency and receives them after backscattering from the target object.

A target can be detected by a single radar if the received signal-to-noise ratio (SNR) $\xi$ is higher than a threshold value $\xi_{\text {th }}$. This SNR threshold depends on the receiver sensitivity, which is the minimum received power $P_{\text {rth }}$ for pulse reception and, consequently, for TOA estimation. The received power from a radar-to-target link of length $r$ is given by

$$
P_{\mathrm{r}}=\int_{f_{\mathrm{L}}}^{f_{\mathrm{U}}} \frac{S_{\mathrm{t}}(f) \eta_{\mathrm{t}}\left(f, \boldsymbol{\Theta}_{\mathrm{t}}\right) \eta_{\mathrm{r}}\left(f, \boldsymbol{\Theta}_{\mathrm{r}}\right) \sigma(f)}{4 \pi r^{2 \beta} L_{\mathrm{d}}^{\prime} 10^{L_{\mathrm{w}}}(f) f^{2 \kappa+2}} d f
$$

where $S_{\mathrm{t}}(f)$ is the transmitted power spectral density (PSD), $\eta_{\mathrm{t}}\left(f, \boldsymbol{\Theta}_{\mathbf{t}}\right)$ and $\eta_{\mathrm{r}}\left(f, \boldsymbol{\Theta}_{\mathbf{r}}\right)$ are the transmitter and receiver antenna gains, $\beta$ is the path loss exponent, $\kappa$ is the frequency decaying exponent, $\left[f_{\mathrm{L}}, f_{\mathrm{U}}\right]$ is the signal bandwidth, and $\sigma(f)$ is the radar cross section (RCS). In (1), the quantity $L_{\mathrm{d}}^{\prime}=L_{\mathrm{d}} d_{0}^{-2 \beta} f_{0}^{-(2 \kappa+2)}$ where $L_{\mathrm{d}}$ is the path loss at the reference distance $d_{0}$ and frequency $f_{0}$, and $L_{\mathrm{w}}(f)$ is the frequency dependent loss in $\mathrm{dB}$ due to the presence of walls and it can be considered zero in LOS conditions. The path loss exponent $\beta$ depends on the environment, varying for LOS or non-line-of-sight (NLOS) propagation. In NLOS propagation, the exponent typically range from 3 to 4 if the path is partially obstructed, and from 4 to 7 for total obstruction $[13,14]$.

From (1), the maximum lenght of radar-to-target link is

$\widehat{r} \triangleq\left(\frac{1}{L_{\mathrm{d}}^{\prime} 4 \pi P_{\mathrm{rth}}} \int_{f_{\mathrm{L}}}^{f_{\mathrm{U}}} \frac{S_{\mathrm{t}}(f) \eta_{\mathrm{t}}\left(f, \boldsymbol{\Theta}_{\mathrm{t}}\right) \eta_{\mathrm{r}}\left(f, \boldsymbol{\Theta}_{\mathrm{r}}\right) \sigma(f)}{10^{L_{\mathrm{w}}}(f) f^{2 \kappa+2}} d f\right)^{1 / 2 \beta}$.

A target can be detected by a radar only if $r \leq \widehat{r} . x f$ Thus, we define the area covered by the $i$ th radar as a circle of radius $\widehat{r}$ centered in $\mathbf{x}_{\mathrm{R}}^{(i)}$, in which TOA can be measured.

We denote with $\boldsymbol{\tau}_{k}=\left[\tau_{k}^{(1)} \tau_{k}^{(2)} \cdots \tau_{k}^{(N)}\right]$ the TOA vector whose elements represent the TOA from each radar $(i=$ $1,2, \ldots, N)$ at the time index $k$. Then, the vector of distances between the target and each radar results in $\mathbf{r}_{k}=$ $\left[r_{k}^{(1)} r_{k}^{(2)} \cdots r_{k}^{(N)}\right]$ where $r_{k}^{(i)}=\tau_{k}^{(i)} c / 2$. Each TOA estimate $\tau_{k}^{(i)}$ can be modeled as

$$
\tau_{k}^{(i)}=\frac{\left\|\mathbf{x}_{k}-\mathbf{x}_{\mathrm{R}}^{(i)}\right\|}{c}+\varepsilon
$$

where the error $\varepsilon$ can be modeled as a Gaussian distributed random variable $\varepsilon \sim \mathcal{N}\left(\mu, \sigma_{\tau}{ }^{2}\right)$ with mean $\mu$ taking into account the presence of positive bias in obstructed paths (zero in LOS conditions) [6]. The standard deviation $\sigma_{\tau}$ depends on the propagation environment, the received SNR $\xi$, and the hardware for TOA estimation. Thus, to analyze WSR network performance the relation between $\sigma_{\tau}$ and $\xi$ is needed. A non-increasing function is typically considered as

$$
\sigma_{\tau}=10^{f\left(\xi_{\mathrm{dB}}\right)}
$$

where $f\left(\xi_{\mathrm{dB}}\right)$ depends on the hardware solution for TOA estimation. In our case study, described in Section 4, energy detector TOA estimation is considered for which $f\left(\xi_{\mathrm{dB}}\right)$ is determined.

The presence of obstacles and walls obstructing signal paths results in a positive bias on the TOA called excess

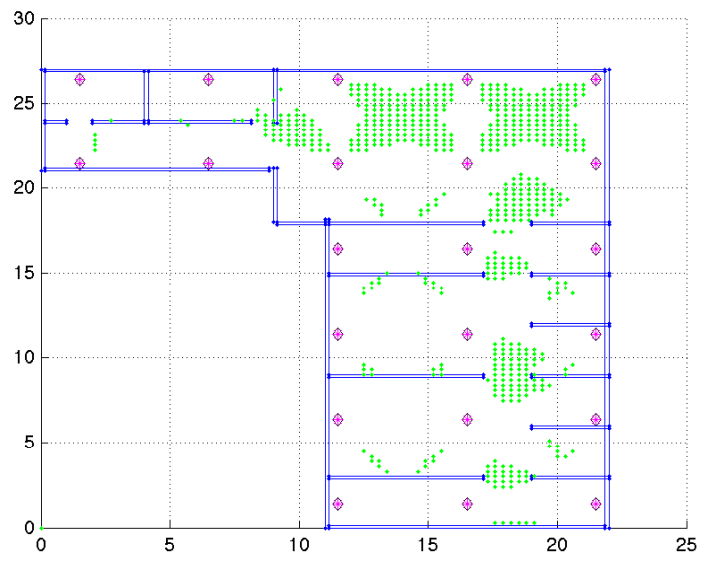

Figure 1: Case study environment, radar positions (magenta) and target positions localized without ambiguities (green).

delay (ED) and in an attenuation called partition penetration loss $[4,7]$. The knowledge of the characteristics of materials is needed to evaluate such effects. Network experiments are performed in [5] also to characterize the ED due to the presence of concrete walls in a typical office building. In particular, for UWB signals, the ED increases with the total thickness of the encountered walls $d_{\mathrm{w}}$ as $d_{\mathrm{w}} / c$. The penetration loss $(\mathrm{dB})$ in $(2)$ due to the presence of walls is given by [4]

$$
L_{\mathrm{w}}(f)=\sum_{j=1}^{n_{\mathrm{tw}}} n_{j} X_{j}(f)
$$

where $n_{\mathrm{tw}}$ is the number of types of walls encountered by the signal, $n_{j}$ is the number of walls of types $j$, and $X_{j}(f)$ is the frequency dependent insertion loss for wall of type $j$.

\section{NAVIGATION ALGORITHM}

The aim of a navigation algorithm is to estimate the target position $\mathbf{x}_{k}$ at each time index $k$ (i.e., the states) from measurements $\mathbf{r}_{k}$ (i.e., the observations) [20]. The Bayesian inference of position's belief $b\left(\mathbf{x}_{k}\right)=p\left(\mathbf{x}_{k} \mid \mathbf{r}_{1: k}\right)$ (i.e., posterior distribution of the position state vector, given past observations) is obtained in two phases: (i) a prediction phase in which the belief is determined based on previous position and a mobility model, and (ii) an update phase where the belief is updated based on new measurements and a perception model. Therefore, by denoting with $\mathbf{r}_{1: k}$ the set of available observations at time index $k, b\left(\mathbf{x}_{k}\right)$ is given by $[1,11]$

$$
b\left(\mathbf{x}_{k}\right)=\frac{b^{-}\left(\mathbf{x}_{k}\right) p\left(\mathbf{r}_{k} \mid \mathbf{x}_{k}\right)}{p\left(\mathbf{r}_{k} \mid \mathbf{r}_{1: k-1}\right)}
$$

where $b^{-}\left(\mathbf{x}_{k}\right)=p\left(\mathbf{x}_{k} \mid \mathbf{r}_{1: k-1}\right)$ is the predicted belief given by

$$
b^{-}\left(\mathbf{x}_{k}\right)=\int p\left(\mathbf{x}_{k} \mid \mathbf{x}_{k-1}\right) b\left(\mathbf{x}_{k-1}\right) d \mathbf{x}_{k-1} .
$$

The term $p\left(\mathbf{x}_{k} \mid \mathbf{x}_{k-1}\right)$ is the mobility model of the target and gives the probability distribution function (PDF) of $\mathbf{x}_{k}$ conditioned on previous position $\mathbf{x}_{k-1}$. The (7) is solved in the prediction phase and then updated according to (6) 
and new measurements. Finally the estimated position $\hat{\mathbf{x}}_{k}$ is determined as that value maximizing $b\left(\mathbf{x}_{k}\right)$.

Various implementations of Bayesian inference are possible, which differ in the beliefs computation. In particular, we consider the PF algorithm. The key idea of PFs is to represent the posterior distribution (the belief), by a set of random samples (particles) with associated weights as

$$
b\left(\mathbf{x}_{k}\right) \approx \sum_{s=1}^{N_{\mathrm{s}}} w_{k, s} \delta\left(\mathbf{x}_{k}-\mathbf{x}_{k, s}\right)
$$

where $N_{\mathrm{s}}$ is the number of particles, $\delta(\cdot)$ is the Delta function, $w_{k, s} \geq 0 \forall k, s$ is the weight for particle $s$ at time index $k$, and $\sum_{s=1}^{N_{\mathrm{s}}} w_{k, s}=1$. The weights are chosen using the principle of importance sampling $[1,9]$ in which a distribution of samples is considered with more dense samples where it is more probable that the object is located. In PF algorithms the main important recursive steps for evaluating the $s$ th particle can be summarized as follow

$$
\begin{array}{rr}
\mathbf{x}_{k, s} \sim p\left(\mathbf{x}_{k} \mid \mathbf{x}_{k-1, s}\right) & \text { mobility model } \\
w_{k, s}=w_{k-1, s} p\left(\mathbf{r}_{k} \mid \mathbf{x}_{k, s}\right) & \text { perception model. }
\end{array}
$$

A Gaussian mobility model is considered as given by

$$
p\left(\mathbf{x}_{k} \mid \mathbf{x}_{k-1}\right)=\frac{1}{\sqrt{2 \pi} \sigma_{\mathrm{m}}} e^{-\frac{\left\|\mathbf{x}_{k}-\mu_{k}\right\|^{2}}{2 \sigma_{\mathrm{m}}^{2}}}
$$

where the standard deviation $\sigma_{\mathrm{m}}$ considers the uncertainty on the target movement, and the mean $\boldsymbol{\mu}_{k}$ depends on $\mathbf{x}_{k-1}$ and intra-node measurements. We propose two different mobility model: the speed known direction unknown (SKDU) and the speed and direction learning (SDL).

In the SKDU model we assume the target speed intensity is known but there are no direction information. The mean of the Gaussian distribution is determined as

$$
\boldsymbol{\mu}_{k}=\mathbf{x}_{k-1}+\mathbf{v} T
$$

where the angle of the speed vector $\mathbf{v}$ is uniformly distributed between $-\pi$ and $\pi$ and $T$ is the time between two consecutive measurements.. In the SDL model the speed vector is completely calculated from previously estimated positions as

$$
\mathbf{v}_{k-1}=\frac{1}{N_{v} T} \sum_{v=1}^{N_{v}}\left(\mathbf{x}_{k-v}-\mathbf{x}_{k-v-1}\right)
$$

where $N_{v}$ is the length of a sliding window of previous states. Hence, the mean of the Gaussian distribution is

$$
\boldsymbol{\mu}_{k}=\mathbf{x}_{k-1}+\mathbf{v}_{k-1} T
$$

We consider independent observations, thus the perception model is given by

$$
p\left(\mathbf{r}_{k} \mid \mathbf{x}_{k}\right)=\prod_{i=1}^{N} p\left(r_{k}^{(i)} \mid \mathbf{x}_{k}\right)
$$

where $r_{k, i}$ is the measurement from the $i$ th radar at time index $k$. We assume a perception model with Gaussian distribution as given by

$$
p\left(r_{k}^{(i)} \mid \mathbf{x}_{k}\right)=\frac{1}{\sqrt{2 \pi} \sigma_{\mathrm{p}}} e^{-\frac{\left(r_{k}^{(i)}-\left\|\mathbf{x}_{k}-\mathbf{x}_{\mathrm{R}}^{(i)}\right\|\right)^{2}}{2 \sigma_{\mathrm{p}}^{2}}} .
$$

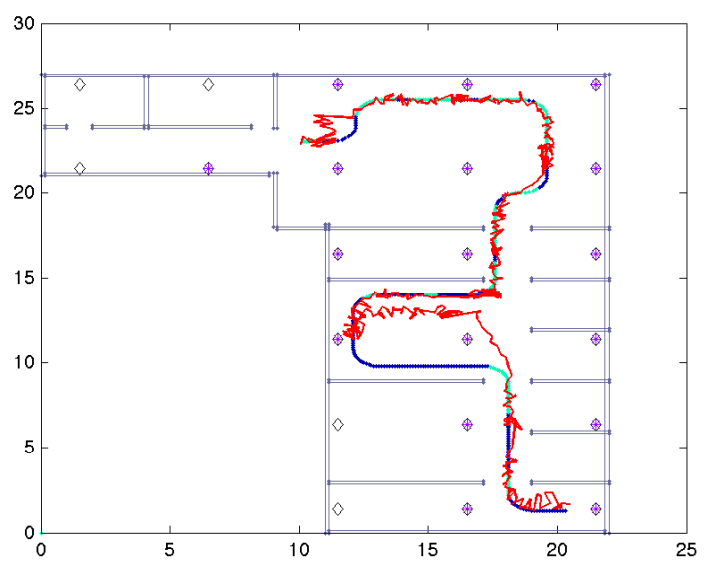

Figure 2: Real and estimated (red line) trajectory with SKDU. Blue and green represent detected only and localized without ambiguities target position, respectively.

The standard deviation $\sigma_{\mathrm{p}}$ depends on both the accuracy of localization technology and signal propagation conditions.

\section{NUMERICAL RESULTS}

We now present navigation performance metrics and results for a case study in indoor environment.

\subsection{Performance Metrics}

As performance metrics we consider the navigation error, the navigation root mean square error (RMSE) and the navigation error outage (NEO). The navigation error, for each time index $k$, is given by

$$
e\left(\mathbf{x}_{k}\right)=\left\|\hat{\mathbf{x}}_{k}-\mathbf{x}_{k}\right\|
$$

which represents the Euclidean distance between the target estimated position $\hat{\mathbf{x}}_{k}$ and the true position $\mathbf{x}_{k}$. From (17), the navigation RMSE results in

$$
e_{\mathrm{RMS}}=\sqrt{\frac{1}{K} \sum_{k=1}^{K}\left[e\left(\mathbf{x}_{k}\right)\right]^{2}} .
$$

where $K$ is the number of the estimated positions along the trajectory. Another important performance metric is the NEO, which is the probability that the navigation error falls below a given target value $e_{\text {th }}$ defined as

$$
\begin{aligned}
P_{\mathrm{NEO}} & =\mathbb{P}\left\{e\left(\mathbf{x}_{k}\right)>e_{\mathrm{th}}\right\} \\
& =\frac{1}{K} \sum_{k=1}^{K} \mathbb{E}\left\{\mathbb{I}\left\{\|\hat{\mathbf{x}}(k)-\mathbf{x}(k)\|>e_{\mathrm{th}}\right\}\right\}
\end{aligned}
$$

where $\mathbb{I}\{\cdot\}$ is the indicator function, which is zero when the proposition is false and one otherwise, and $\mathbb{E}\{\cdot\}$ represents the spatial-temporal statistical expectation.

\subsection{Case study}

We now provide results for a monostatic WSR in the environment of Fig. 1. The WSR is composed of 22 UWB monostatic radars located at the corner of squares with side of 5 


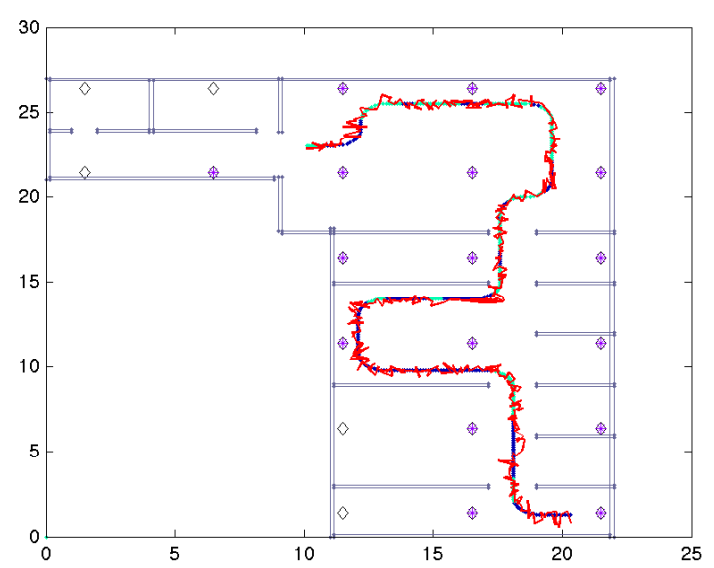

Figure 3: Real and estimated (red line) trajectory with SDL. Blue and green represent detected only and localized without ambiguities target position, respectively.

m. The UWB transmitted PSD is $S_{\mathrm{t}}=-42 \mathrm{dBm} / \mathrm{MHz}$ over the $1.7 \mathrm{GHz}$ frequency bandwidth $\left(f_{\mathrm{L}}=3.1 \mathrm{GHz}, f_{\mathrm{U}}=4.8\right.$ $\mathrm{GHz}$ ), which is the European UWB lower band. We consider coherent accumulation of 126 pulses with frequency of pulse repetition $10 \mathrm{MHz}$ for obtaining a TOA estimation, omnidirectional antennas, $\eta_{\mathrm{t}}\left(f, \boldsymbol{\Theta}_{\mathbf{t}}\right)=\eta_{\mathrm{r}}\left(f, \boldsymbol{\Theta}_{\mathbf{r}}\right)=1$, and RCS $\sigma(f)=1 \mathrm{~m}^{2}$. The reference distance $d_{0}$ is set to $1 \mathrm{~m}, \kappa=0$, and $L_{0}=c^{2} /\left(4 \pi f_{0}\right)^{2}$ where $f_{0}$ is the central frequency. The path loss exponent $\beta$ has been chosen equal to 3.07 (i.e., NLOS condition for the standard IEEE 802.15.4a) despite that we consider also the effects of each wall. This choice allows a worst-case performance evaluation. The minimum receiver sensitivity is set to $P_{\text {rth }}=-85 \mathrm{dBm}$. Note that with the chosen parameters a target located in any position of the SA can be detected. The target positions where localization is possible without ambiguities (i.e., at least three radars detect the target) is also shown in Fig. 1.

As discussed in Section 2, a key aspect affecting the performance is the ability of accurate TOA estimation for a given received SNR which is modeled by function $f\left(\xi_{\mathrm{dB}}\right)$ in (4). This function has been chosen following a characterization activity on energy detector based TOA estimation. In this case, it is possible to distinguish three different SNR regions. The regions of small and large SNR values respectively correspond to regions of large and small constant standard deviation $\sigma_{\tau}$. A transition region is verified for moderate SNR values. In particular, the upper and lower values of $\sigma_{\tau}$ depend on the observation time $T_{\mathrm{g}}$ and the integration time $T_{\text {int }}$ of the energy detector, respectively, as follows

$$
f\left(\xi_{\mathrm{dB}}\right)= \begin{cases}\log _{10}\left(\frac{T_{\mathrm{g}}}{\sqrt{12}}\right) & \xi_{\mathrm{dB}} \leq \xi_{\mathrm{dB}}^{(\mathrm{L})} \\ \mathrm{A}+\mathrm{B} \xi_{\mathrm{dB}} & \xi_{\mathrm{dB}}^{(\mathrm{L})}<\xi_{\mathrm{dB}} \leq \xi_{\mathrm{dB}}^{(\mathrm{H})} \\ \log _{10}\left(\frac{T_{\mathrm{int}}}{\sqrt{12}}\right) & \xi_{\mathrm{dB}}>\xi_{\mathrm{dB}}^{(\mathrm{H})}\end{cases}
$$

For our case study we consider $T_{\mathrm{int}}=4 \mathrm{~ns}, T_{\mathrm{g}}=120 \mathrm{~ns}$, $\mathrm{A}=-7.39, \mathrm{~B}=-0.0875, \xi_{\mathrm{dB}}^{(\mathrm{L})}=0.8 \mathrm{~dB}$, and $\xi_{\mathrm{dB}}^{(\mathrm{H})}=17 \mathrm{~dB}$. To account for the effects of walls in the environment propagation, we choose the trajectory shown in Fig. 2 where two different situations can be distinguished. In the first

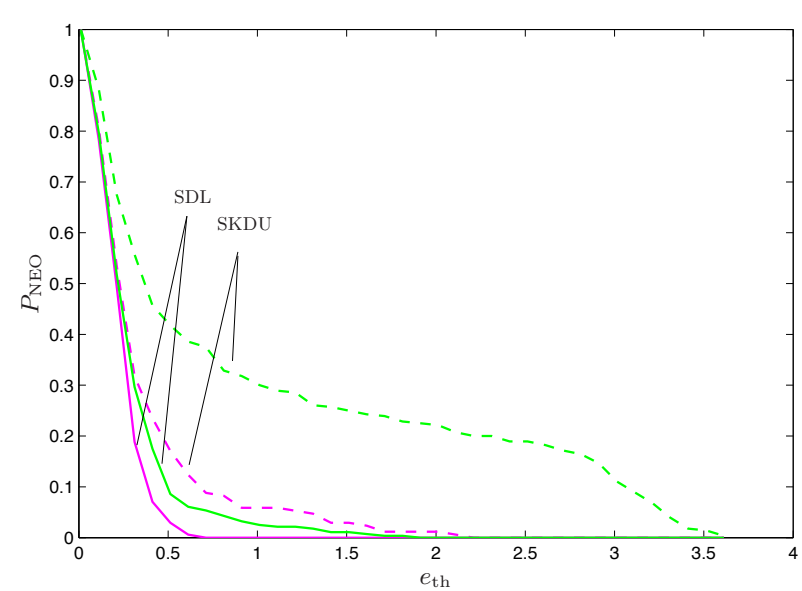

Figure 4: NEO for SKDU (dashed) and SDL (solid) in LOS (magenta) and OLOS (green) conditions.

part of the trajectory, no walls obstruct the signal path between the target and the radars able to detect it, while in the second part OLOS conditions are present. The velocity of the target is $v=1 \mathrm{~m} / \mathrm{sec}$, the time between two consecutive measurements is $T=0.1 \mathrm{~s}$ and for the perception model $\sigma_{\mathrm{p}}=0.2 \mathrm{~m}$ has been considered. Figure 2 shows the real and the estimated trajectories when considering the SKDU mobility model with $\sigma_{\mathrm{m}}=0.8 \mathrm{~m}$. This value is such that with probability 0.9 the new position is within a circle centered in $\mu_{k}$ of radius $v T$, with $v$ the speed we assumed known. The two trajectories can be compared in both detection only and localization without ambiguities conditions. In Fig. 3 similar results are shown for the SDL case with sliding window $N_{v}=10$, and standard deviation calculated as for SKDU with $v=\left|\mathbf{v}_{k-1}\right|$. The RMSE for the SKDU is $1.2 \mathrm{~m}$, whereas for the SDL is $0.35 \mathrm{~m}$. This results show a clear difference, in terms of global navigation accuracy over the trajectory between the two mobility models. Moreover, note that the estimated trajectory shown in Fig. 2 for the SKDU mobility model presents an ambiguity which is solved by means of SDL mobility model in Fig. 3. Finally, in Fig. 4 the NEO is given for SKDU and SDL mobility models for the two parts of the trajectory in LOS and OLOS conditions. The figure shows as the mobility model and the propagation conditions strongly impact the NEO. For example, results in Fig. 4 indicate that using SKDU in $80 \%$ of cases the navigation error is below $0.5 \mathrm{~m}$ in the first part of the trajectory (LOS) and it is below $2.7 \mathrm{~m}$ in the second part (OLOS). When SDL is used, in $80 \%$ of cases the navigation error is below $0.3 \mathrm{~m}$ in the first part of the trajectory (LOS) and it is below $0.4 \mathrm{~m}$ in the second part (OLOS).

\section{CONCLUSION}

A framework for analysis and design of monostatic WSR networks for passive localization and navigation is presented. The framework accounts for the network setting, environment propagation, TOA estimation techniques, and Bayesian navigation algorithms based on perception and mobility models. Navigation techniques based on particle filter algorithm and different mobility models have been compared in terms of localization error and navigation error outage for a case 
study in indoor environments. It is shown as mobility model can enhance navigation performance even in difficult environment propagation.

\section{Acknowledgments}

This research was supported, in part, by the European Commission in the scope of the FP7 ICT project SELECT Grant no.257544 and the Italian Ministero dello Sviluppo Economico under the project WEBS. Authors thank A.Giorgetti for helpful discussions.

\section{REFERENCES}

[1] M. S. Arulampalam, S. Maskell, N. Gordon, and T. Clapp. A tutorial on particle filters for online nonlinear/non-Gaussian Bayesian tracking. IEEE Trans. Signal Process., 50(2):174-188, Feb. 2002.

[2] S. Bartoletti and A. Conti. Passive network localization via UWB wireless sensor radars: the impact of TOA estimation. In Proc. IEEE Int. Conf. on Ultra-Wideband (ICUWB), pages 576-580, Bologna, Italy, Sept. 2011.

[3] S. Bartoletti, A. Conti, and A. Giorgetti. Analysis of UWB radar sensor networks. In Proc. IEEE Int. Conf. on Commun., Cape Town, South Africa, May 2010.

[4] R. Buehrer, W. Davis, and A. S.-J. D. Sweeney. Ultra-wideband propagation measurements and modeling, darpa netex final technical report, jan. 2004. DARPA NETEX Final Technical Report, Jan. 2004.

[5] A. Conti, M. Guerra, D. Dardari, N. Decarli, and M. Z. Win. Network experimentation for cooperative localization. IEEE J. Sel. Areas Commun., 30, 2012. to appear.

[6] D. Dardari, A. Conti, U. J. Ferner, A. Giorgetti, and M. Z. Win. Ranging with ultrawide bandwidth signals in multipath environments. Proc. IEEE, 97(2):404-426, Feb. 2009. special issue on Ultra-Wide Bandwidth (UWB) Technology $\&$ Emerging Applications.

[7] D. Dardari, A. Conti, J. Lien, and M. Z. Win. The effect of cooperation on localization systems using UWB experimental data. EURASIP J. Appl. Signal Process. (special issue on Cooperative Localization in Wireless Ad Hoc and Sensor Networks), 2008:Article ID 513873, 1-11, 2008.

[8] D. Dardari, R. D'Errico, C. Roblin, A. Sibille, and M. Z. Win. Ultrawide bandwidth RFID: The next generation? Proc. IEEE, 99(7):1570-1582, July 2010. special issue on RFID - A Unique Radio Innovation for the 21st Century.

[9] R. Douc and O. Cappe. Comparison of resampling schemes for particle filtering. In Image and Signal Processing and Analysis, 2005. ISPA 2005. Proceedings of the 4th International Symposium on, pages $64-69$, Sept. 2005.

[10] S. Gezici, Z. Tian, G. B. Giannakis, H. Kobayashi, A. F. Molisch, H. V. Poor, and Z. Sahinoglu. Localization via ultra-wideband radios: a look at positioning aspects for future sensor networks. IEEE Signal Process. Mag., 22(4):70-84, July 2005.

[11] F. Gustafsson, F. Gunnarsson, N. Bergman, U. Forsell, J. Jansson, R. Karlsson, and P. J. Nordlund. Particle filters for positioning, navigation and tracking. IEEE Trans. Signal Process., 50(2):425-437, Feb. 2002.

[12] A. Haimovich, R. Blum, and L. Cimini. MIMO radar with widely separated antennas. Signal Processing Magazine, IEEE, 25(1):116 -129, Jan. 2008.

[13] A. F. Molisch. Ultra-wide-band propagation channels. Proc. IEEE, 97(2):353-371, Feb. 2009.

[14] A. F. Molisch, K. Balakrishnan, C. chin Chong, S. Emami, A. Fort, J. Karedal, J. Kunisch, H. Schantz, U. Schuster, and K. Siwiak. IEEE 802.15.4a channel model - final report. Tech. Rep. Document IEEE 802.15-04-0662-02-004a, Nov. 2004

[15] A. F. Molisch, D. Cassioli, C.-C. Chong, S. Emami, A. Fort, B. Kannan, J. Karedal, J. Kunisch, H. Schantz, K. Siwiak, and M. Z. Win. A comprehensive standardized model for ultrawideband propagation channels. IEEE Trans. Antennas Propag., 54(11):3151-3166, Nov. 2006. special issue on Wireless Communications.

[16] E. Paolini, A. Giorgetti, M. Chiani, R. Minutolo, and M. Montanari. Localization capability of cooperative anti-intruder radar systems. EURASIP Journal on Advances in Signal Processing, Special Issue on Cooperative Localization in Wireless Ad Hoc and Sensor Networks, pages 1-14, Mar. 2008.

[17] L. Reggiani and R. Morichetti. Hybrid active and passive localization for small targets. In Indoor Positioning and Indoor Navigation (IPIN), 2010 International Conference on, pages $1-5$, Sept. 2010.

[18] Y. Shen and M. Z. Win. Fundamental limits of wideband localization - Part I: A general framework. IEEE Trans. Inf. Theory, 56(10):4956-4980, Oct. 2010.

[19] Y. Shen, H. Wymeersch, and M. Z. Win. Fundamental limits of wideband localization - Part II: Cooperative networks. IEEE Trans. Inf. Theory, 56(10):4981-5000, Oct. 2010.

[20] M. Z. Win, A. Conti, S. Mazuelas, Y. Shen, W. M. Gifford, D. Dardari, and M. Chiani. Network localization and navigation via cooperation. IEEE Commun. Mag., 49(5):56-62, May 2011.

[21] M. Z. Win and R. A. Scholtz. Impulse radio: How it works. IEEE Commun. Lett., 2(2):36-38, Feb. 1998.

[22] M. Z. Win and R. A. Scholtz. Ultra-wide bandwidth time-hopping spread-spectrum impulse radio for wireless multiple-access communications. IEEE Trans. Commun., 48(4):679-691, Apr. 2000.

[23] M. Z. Win and R. A. Scholtz. Characterization of ultra-wide bandwidth wireless indoor communications channel: A communication theoretic view. IEEE J. Sel. Areas Commun., 20(9):1613-1627, Dec. 2002.

[24] L. Yang and G. B. Giannakis. Ultra-wideband communications: An idea whose time has come. IEEE Signal Process. Mag., 21(6):26-54, Nov. 2004.

[25] Y. Zhou, C. L. Law, Y. L. Guan, and F. Chin. Localization of passive target based on UWB backscattering range measurement. In Proc. IEEE Int. Conf. on Ultra-Wideband (ICUWB 2009), pages 145-149, Vancouver, CANADA, Sept. 2009. 